\title{
OBSERVATIONAL CONSTRAINTS ON SILICON CHEMISTRY IN THE CIRCUMSTELLAR ENVELOPES OF RED GIANTS
}

\author{
Raghvendra Sahai \\ Millimeter Wave Observatory \\ Department of Astronomy \\ University of Texas \\ Austin, TX 78712
}

\begin{abstract}
We review observations to date of silicon-bearing molecules in circumstellar gas and dust around red giants. New constraints on circumstellar chemistry are provided by the discovery of (i) a systematic decrease in the $[\mathrm{SiO}] /\left[\mathrm{H}_{2}\right]$ ratio with mass loss rate, ii) $\mathrm{SiO}$ in carbon-rich CIT 6. The presence/absence of SiO maser emission in oxygen-rich/carbon-rich envelopes is linked to differences in grain composion and the grain-condensation radius.
\end{abstract}

The presence of silicon in the circumstellar envelopes of red giants is manifest from observations of millimeter emission from rotational lines of the molecules $\mathrm{SiO}$ and SiS. Certain features in the infrared spectra of these objects are attributed to siliconbearing grains. Our current understanding of silicon chemistry in these objects, based in large part on these observations, may be summarised as follows: in oxygen rich stars $(\mathrm{O} / \mathrm{C}>1) \mathrm{Si}$ combines with $\mathrm{O}$ somewhere in the cool red giant atmosphere to form SiO, and is carried along with the general molecular outflow into the circumstellar domain; in carbon rich stars $(\mathrm{C} / \mathrm{O}>1)$, SiS and $\mathrm{SiO}$ constitute the major molecular carriers of silicon. The SiO abundance in the outer envelopes $\left(>10^{15} \mathrm{~cm}\right)$ of oxygen rich stars, as deduced from non-maser V=0, J=2-1 lines (Morris, M. and Alcock, C. A. 1977, Ap. J., 218, 687 ) is found to be typically $2-3$ orders of magnitude less than that expected in the region of formation from chemical equilibrium (Tsuji, T. 1973, Astr. Ap., 23, 411).

Observational indications that the $\mathrm{SiO}$ abundance at formation is indeed high (roughly equal to the cosmic abundance of silicon) come from the very large intensities of the $\mathrm{SiO}$ maser lines seen in these objects. The presence of emission or absorption features at 10 microns from these sources is attributed to 'silicate' dust (Woolf, N. J. and Ney, E. P. 1969, Ap. J. (Letters), 155, L181; Gehrz, R. D. and Woolf, N. J. 1971, Ap. J., 165, 285 ), indicating that circumstellar grains play an important role in depleting $\mathrm{SiO}$ (and $\mathrm{Si}$ bearing species in general). In carbon-rich stars, the SiS abundance in the extended envelope (Sahai, R., Wootten, A., and Clegg, R. E. S. 1984 Ap. J., 284, 144, hereafter SWC) is less by roughly 2 orders of magnitude than the value expected from chemical modelling (McCabe, E. M., Smith, R. C., and Clegg, R. E. S. 1979, Nature, 281, 263). SWC show that this deficit results mainly from $\mathrm{Si}$, and not $\mathrm{S}$ depletion. An 11 micron emission feature seen in the infrared spectra of many carbon stars, identified as emission from condensed SiC (Treffers, R. and Cohen, M. 1974, Ap.J., 188, 545), suggests that $\mathrm{Si}$ is depleted by $\mathrm{SiC}$ grain formation in these objects.

The $\mathrm{SiO}$ abundance depends on the $\mathrm{C} / \mathrm{O}$ ratio in the red giant atmosphere. In oxygen rich stars $\mathrm{SiO}$ is predicted to be the major silicon bearing molecule. The presence 
of both maser and non-maser $\mathrm{SiO}$ emission from a sizable number of oxygen rich stars, and the absence of SiS (SWC) indicates that SiO is indeed the dominant silicon species in these objects. For carbon rich objects $\mathrm{SiS}$ is expected to be more abundant than $\mathrm{SiO}$ by a moderate factor $(<10)$ which depends both on the elemental $\mathrm{C} / \mathrm{O}$ and the $\mathrm{Si} / \mathrm{S}$ ratio in the red giant atmosphere. The observational evidence for such a chemistry is more scanty: only in one object, IRC+10216, non-maser (but not maser) SiO emission, as well as SiS emission, has been observed, and the $\mathrm{SiS} / \mathrm{SiO}$ abundance ratio is found to be larger than unity. SWC have found SiS emission from three other carbon rich stars, but no SiO measurements have been reported for these or any other carbon stars. In this paper, we add three new results which further constrain silicon chemistry in red giant envelopes.

i) The depletion of $\mathrm{SiO}$ increases systematically with $M$, the mass loss rate (Figure). The decrease of the fractional abundance, $\mathrm{f}(\mathrm{SiO})=$ $[\mathrm{SiO}] /\left[\mathrm{H}_{2}\right]$, with $\mathrm{M}$ is found to obey a power law relationship. This result is obtained from a deduction of $\mathrm{f}(\mathrm{SiO})$ from observed $\mathrm{SiO} \mathrm{V}=0, \mathrm{~J}=2-1$ line intensities using the models of Morris and Alcock (1977), together with $M$ derived from $\mathrm{CO} \mathrm{J}=1-0$ observations by Knapp and Morris (1985, Ap.J., 292,640 ). It is in conflict with the theoretical model of grain condensation in oxygen-rich red giants given by Deguchi (1980, Ap. J., 236, 567), which predicts that an increase in the mass loss rate should result in an increase in the $[\mathrm{SiO}] /\left[\mathrm{H}_{2}\right]$ ratio.

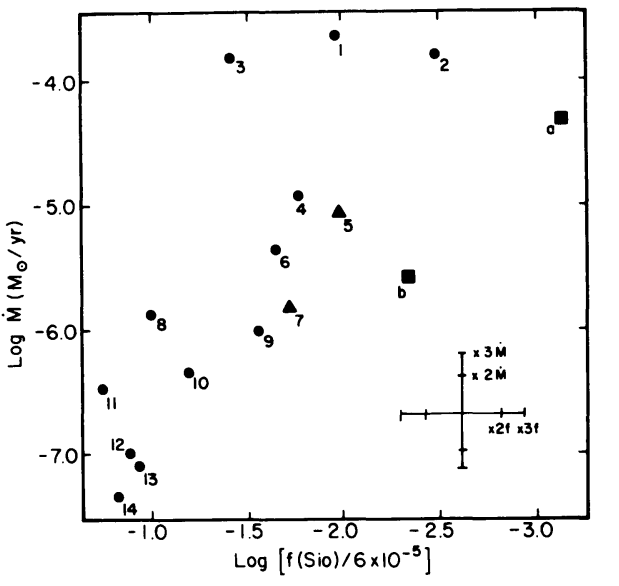

$$
\begin{aligned}
& \text { Oxygen Rich \& S-stors } \\
& 1=V Y \text { Cmo } \quad 2=\text { NML Cyg } \quad 3=I R C 10420 \quad 4=\mid R C 10011 \quad 5=W \text { AqI (S) } \\
& 6=\text { IK Tou } 7=\text { Chi Cyg (S) } 8=R T \text { Vir } \quad 9=R \text { Aql } \quad 10=R \text { Cos } \\
& 11=\operatorname{RxB} B 00 \quad 12=\text { Wyo } \quad 13 \times R \text { LeO } 14=R \text { Hyo } \\
& \text { Corbon Rich Stors } \\
& 0=\operatorname{IRC} 10216 \quad b=\text { Cit } 6
\end{aligned}
$$

ii) We report the first detection of $\mathrm{SiO}$ in CIT 6, a carbon rich object, in both the $\mathrm{V}=0, \mathrm{~J}=3-2$ and the $\mathrm{J}=5-4$ lines (Sahai, R., Wannier, P. G., and Claussen, M. J. 1985, in preparation). This is the second carbon rich object after IRC+10216 in which SiO has been detected. The SiS/SiO abundance ratio in CIT 6 is probably larger than unity but smaller than in IRC +10216 . These conclusions are tentative, since the SiS abundance in CIT 6 is determined from a single rotational line with poor signal to noise.

iii) We suggest a possible explanation for the fact that $\mathrm{SiO}$ maser emission is prolific among oxygen rich stars, but is not observed in the carbon rich stars, IRC+10216 and CIT 6, even though non-maser SiO emission from both carbon rich and oxygen rich stars is of comparable intensity. We propose that the formation of SiC grains in carbon-rich objects depletes enough silicon sufficiently close to the star, such that the abundance of $\mathrm{SiO}$ is never large enough for observable masering to be detected. In oxygen rich stars, however, silicon is depleted by the formation of silicates, which have a lower condensation temperature than $\mathrm{SiC}$, and form further out in the circumstellar envelope, beyond the region where the masers are formed. This conclusion is also supported by the moderate [SiS]/[SiO] ratio of $<5$ in IRC +10216 and CIT 6 , which can occur only at fairly low equilibrium temperatures of $<1150^{\circ} \mathrm{K}$, suggesting that $\mathrm{SiS}$ and $\mathrm{SiO}$ form after/during, but not before the condensation of $\mathrm{SiC}$ grains which occurs at $1300^{\circ} \mathrm{K}$. 